\title{
Reliability of Reconstructed Breast Flap after Chemotherapy and Radiotherapy in Immediate Breast Reconstruction
}

\author{
Keun-Cheol Lee ${ }^{1}$, Tae-Heon Kim ${ }^{1}$, Su-Seong Park ${ }^{1}$, Min-Su Kim ${ }^{1}$, Myung-Hoon Kim ${ }^{1}$, \\ Seok-Kwun Kim ${ }^{1}$, Se-Heon $\mathrm{Cho}^{2}$, Mi-Ri Lee ${ }^{2}$, Jin-Hwa Lee ${ }^{3}$, Hyung-Sik Lee ${ }^{4}$, Dae-Cheol Kim ${ }^{5}$ \\ Departments of ${ }^{1}$ Plastic and Reconstructive Surgery, ${ }^{2}$ General Surgery, ${ }^{3}$ Radiology, ${ }^{4}$ Radiation Oncology, and ${ }^{5}$ Pathology, Dong-A University \\ College of Medicine, Busan, Korea
}

Background Postmastectomy adjuvant therapy is used to prevent locoregional recurrence and improve overall breast cancer specific survival rates. However, it can adversely affect the cosmetic results of reconstruction. Therefore, the authors examined flap stability and patients' satisfaction with immediate breast reconstruction after adjuvant therapy.

Methods We retrospectively reviewed the medical records of 204 patients from January 2006 to November 2011. For complication rates, the authors categorized the patients who underwent the immediate breast reconstruction into 4 groups: adjuvant chemotherapy and radiotherapy group, adjuvant chemotherapy only group, adjuvant radiotherapy only group, and the group that did not undergo adjuvant therapy. For comparison of patients' satisfaction, the study was performed with an additional 16 patients who had undergone delayed breast reconstruction.

Results Regarding complication rates, the group that had undergone adjuvant therapy showed no significant difference compared to the group that did not undergo adjuvant therapy. In evaluating the patients' satisfaction, there was no significant difference.

Conclusions Even after adjuvant therapy, immediate breast reconstruction showed good results with respect to flap stability and patients' satisfaction. Immediate breast reconstruction and adjuvant therapy is a safe and useful option for breast cancer patients.

Keywords Drug therapy / Radiotherapy / Breast / Surgical flaps
Correspondence: Keun-Cheol Lee Department of Plastic and Reconstructive Surgery, Dong-A University College of Medicine, 26 Daesingongwon-ro, Seo-gu, Busan 602-715, Korea

Tel: +82-51-240-2968

Fax: +82-51-243-5416 E-mail: pokdungi@dau.ac.kr

This study was supported by research funds from Dong-A university.

This study was presented at the 10th Korea-Japan Congress of Plastic and Reconstructive Surgery, on June 16-18, 2010 in Busan, Korea.

No potential conflict of interest relevant to this article was reported.

Received: 1 Apr 2012 • Revised: 24 Jul 2012 • Accepted: 25 Jul 2012

pISSN: 2234-6163 • elSSN: 2234-6171 • http://dx.doi.org/10.5999/aps.2012.39.5.497• Arch Plast Surg 2012;39:497-503

\section{INTRODUCTION}

The recent increase in the incidence of breast cancer worldwide and in Korea has made the disease the most common in women since 2001.

Breast reconstruction not can enhance the body image of women but also can have a positive effect on sexual and social factors in a woman's life [1]. Among the cases of breast reconstruction surgery, the number of cases of immediate breast reconstruction surgery has recently increased significantly because the procedure can reduce the patient's shock when seeing herself after mastectomy and can reduce medical costs because the surgery is performed only once. Among the cases of mastectomy, performing immediate breast reconstruction after skin-

Copyright $($ C 2012 The Korean Society of Plastic and Reconstructive Surgeons

This is an Open Access article distributed under the terms of the Creative Commons Attribution Non-Commercial License (http://creativecommons.org/

licenses/by-nc/3.0/) which permits unrestricted non-commercial use, distribution, and reproduction in any medium, provided the original work is properly cited.

www.e-aps.org 
sparing mastectomy has been shown to be cosmetically superior to radical mastectomy or modified radical mastectomy, and various long-term studies have shown that locoregional recurrence or complications of cancer have not increased as significantly as with radical mastectomy. Regarding locoregional mastectomy and skin-sparing mastectomy, skin-sparing mastectomy is attempted when breast-conserving surgery cannot be performed because malignant calcified deposits have spread to a wide area of the breast, or several cancers are located at the center of the breast, around the nipples. After performing any of these procedures, adjuvant therapy will be essential for preventing locoregional recurrence or metastasis. While adjuvant therapy after mastectomy has the advantages of preventing locoregional recurrence of breast cancer and improving the average survival rate [1-5], it can cause various complications with respect to the stability of the flaps [2]. Whether performing adjuvant therapy after immediate breast reconstruction, in contrast to delayed breast reconstruction, has an impact on flap stability remains under dispute. In studies up to the present, delayed breast reconstruction has had a lower incidence of complications from postoperative chemotherapy or radiotherapy than immediate breast reconstruction; hence, delayed breast reconstruction has been recommended over immediate breast reconstruction $[2,3]$.

In this study, the authors report on the relatively satisfactory results of the survey they conducted on the stability of flaps and patient satisfaction with adjuvant chemotherapy and radiotherapy after undergoing immediate breast reconstruction at the authors' hospital.

\section{METHODS}

\section{Patients}

This study consisted of 204 patients who underwent immediate breast reconstruction due to breast cancer at the authors' hospital from January 2006 to November 2011. The average age of the patients was 47.1 years, and the follow-up period lasted 1 to 68 months, with an average of 35.1 months. Among these patients, 183 patients underwent postoperative adjuvant chemotherapy or radiotherapy after immediate breast reconstruction following mastectomy; the other 21 patients did not undergo adjuvant therapy after immediate breast reconstruction.

\section{Methods}

Information was collected through chart reviews and interviews with the patients. To analyze the incidence of complications, the authors categorized the patients who underwent the immediate breast reconstruction into 4 groups: the adjuvant chemotherapy and radiotherapy group; adjuvant chemotherapy only group; adjuvant radiotherapy only group; and no adjuvant therapy group. The incidence of complications was investigated via periodic follow-ups through physical examinations, routine mammography, magnetic resonance imaging (MRI), and ultrasonography. The authors also analyzed the patients' satisfaction by comparing the results between the adjuvant therapy only group and the no adjuvant therapy group. For comparison of the patients' satisfaction only, the authors surveyed 16 patients who underwent delayed breast reconstruction during the same period. Of those, 10 patients had undergone adjuvant therapy after total mastectomy. All of the patients underwent delayed breast reconstructive surgery using transverse rectus abdominis myocutaneous (TRAM) pedicled flap. The patients' satisfaction was assessed through surveys using the BREAST-Q module, along with the assessment of the subjective satisfaction of the surgeon.

\section{Statistical analysis}

Statistical analysis was conducted using the non-parametric test. Whether there was a significant difference in the incidence of complications between the subject group and each control group was verified using Fisher's chi-squared test. For the analysis of patient satisfaction in each of the control groups, Wilcoxon's ranksum test was performed by averaging the scores for each item of the questionnaire. The difference was defined as statistically significant when the P-value was below 0.05 .

\section{RESULTS}

A total of 204 patients underwent immediate breast reconstructive surgery using autologous tissue. Among them, 174 patients underwent breast reconstructive surgery using latissimus dorsi (LD) pedicled flap, and 30 patients underwent TRAM pedicled flap. The group using LD pedicled flap showed 17 (9.7\%) complications and a 3.12 satisfaction score. The group using TRAM pedicled flap showed 4 (13.3\%) complications and a 3.13 satisfaction score. According to the surgical method, the incidence of complications and patients' satisfaction are shown in Table 1.

According to the preoperative stage, 37 patients were in stage 0,91 patients were in stage I, 55 patients were in stage IIA, 10 patients were in stage IIB, 4 patients were in stage IIIA, 3 pa-

Table 1. Surgical methods of immediate breast reconstruction

\begin{tabular}{|lccc|}
\hline Surgical methods & $\begin{array}{c}\text { Complica- } \\
\text { tions (\%) }\end{array}$ & $\begin{array}{c}\text { Satisfaction } \\
\text { score }\end{array}$ & Numbers \\
\hline LD pedicled flap & $17(9.7)$ & 3.12 & 174 \\
TRAM pedicled flap & $4(13.3)$ & 3.13 & 30 \\
Total & $21(10.2)$ & - & 204 \\
\hline LD, latissimus dorsi; TRAM, transverse rectus abdominis myocutaneous. \\
\hline
\end{tabular}


tients were in stage IIIB, and 4 patients were in stage IIIC. The amount of excision according to the each stage was $197.5 \mathrm{~g}$ in stage $0,118.4 \mathrm{~g}$ in stage I, $177.6 \mathrm{~g}$ in stage IIA, $260 \mathrm{~g}$ in stage IIB, $134.3 \mathrm{~g}$ in stage IIIA, $145 \mathrm{~g}$ in stage IIIB, and $120.6 \mathrm{~g}$ in stage IIIC (Table 2).

Among the 204 patients, 101 patients underwent both chemotherapy and radiotherapy, postoperatively, 22 patients underwent postoperative chemotherapy only, and 60 patients underwent postoperative radiotherapy only; 21 patients did not undergo adjuvant therapy after immediate breast reconstruction (Table 3 ).

A total of 123 patients underwent postoperative chemotherapy and began therapy on average 24.4 days after breast reconstruction, and there was no difference between this average number of days before the start of the therapy and that of the group that did not undergo the chemotherapy without breast reconstruction after mastectomy. The patients were treated with a combination of adriamycin (or epirubicin) and cyclophosphamide, administered in 4 cycles. There were differences, however, in chemotherapy according to the results of the sentinel lymph node biopsies. Taclipaxel was administered in 4 cycles in the group that had positive sentinel lymph node biopsy results, but there was no increase in the incidence of special complications in the patient group that received additional therapy.

Radiotherapy was performed on a total of 161 patients. The level of radiation on the entire breast was 5,040 to 6,040 cGY. This therapy was administered 22 times on 150 patients and 28 times on 11 patients. Radiotherapy was administered an average of 32.3 days, which did not significantly differ from that of the

Table 2. Number of patients and the weight of the resected tumor in each stage

\begin{tabular}{|lcc|}
\hline Stage & Numbers & $\begin{array}{c}\text { Weight of the excised } \\
\text { mass }(\mathbf{g})\end{array}$ \\
\hline 0 & 37 & 197.5 \\
I & 91 & 118.4 \\
Ila & 55 & 177.6 \\
IIb & 10 & 260 \\
IIla & 4 & 134.3 \\
IIIb & 3 & 145 \\
IIIc & 4 & 120.6 \\
\hline
\end{tabular}

Table 3. Classification according to the differences in adjuvant therapy after immediate breast reconstruction

\begin{tabular}{|lc|}
\hline Adjuvant therapy & Number \\
\hline Chemotherapy + radiotherapy & 101 \\
Chemotherapy only & 22 \\
Radiotherapy only & 60 \\
No adjuvant therapy & 21 \\
Total & 204 \\
\hline
\end{tabular}

group that underwent radiotherapy without breast reconstruction. Among the patients who underwent breast reconstruction, an additional 1,000 cGY of radiotherapy was performed on the primary lesion according to the size of the tumor, the conditions that surrounded the lumpectomy, and the surgical method. It was performed to prevent the locoregional recurrence of the primary lesion due to the greater risk of recurrence with partial mastectomy.

Among the total of 183 patients that underwent postoperative adjuvant therapy, 162 (88.6\%) patients had no complication after adjuvant therapy (Figs. 1-3), and 21 (11.4\%) patients had complications. Of these, 4 patients (2.4\%) had redness (Fig. 4); 4 (2.4\%), swelling; 5 (2.3\%), flap hardening; 4 (1.8\%), hyperpigmentation (Fig. 5); 4 (1.8\%), fat necrosis; 1 (0.6\%), bullae;

\section{Fig. 1. No complications after adjuvant therapy with RTx}

A 49-year-old woman who underwent breast reconstruction with latissimus dorsi pedicled flap; 6,040 cGY of radiotherapy was administered. (A) Preoperative view. (B) Post-adjuvant therapy at the 5 year follow-up. There was no complication after radiotherapy. RTx, radiotherapy.
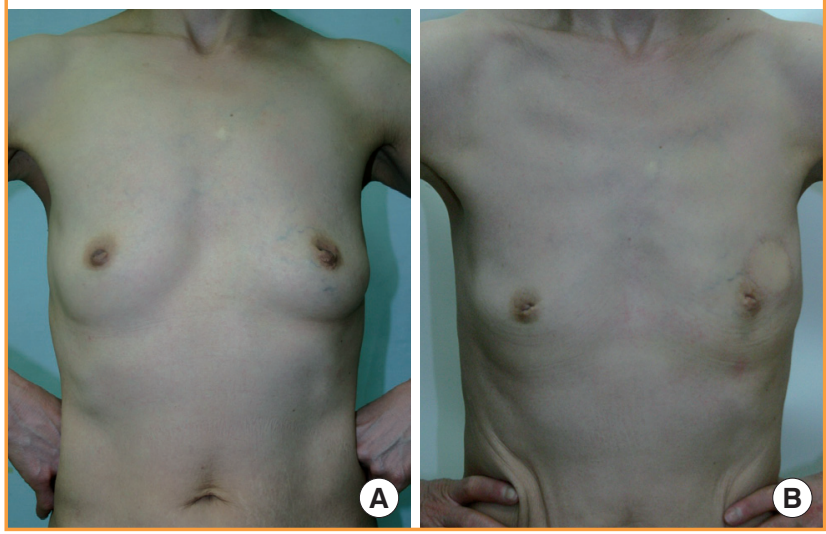

Fig. 2. No complication after adjuvant therapy with CTx+RTx

A 53-year-old woman who underwent breast reconstruction with latissimus dorsi pedicled flap. Chemotherapy was administered 4 times along with 6,040 cGY of radiotherapy. (A) Preoperative view. (B) Postadjuvant therapy at the 2-year follow-up. There was no complication after radiotherapy. CTx, chemotherapy; RTX, radiotherapy.
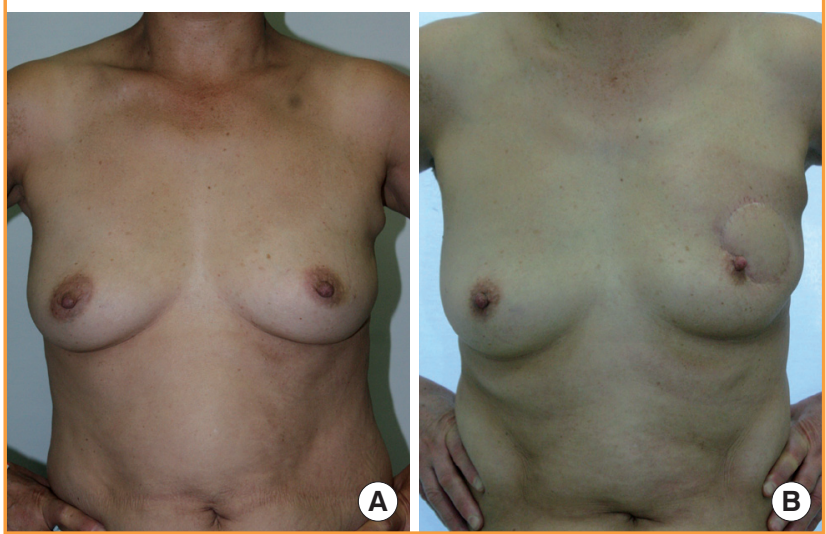


\section{Fig. 3. No complication after adjuvant therapy with CTx+RTx}

A 40-year-old woman who underwent breast reconstruction with transverse rectus abdominis myocutaneous pedicled flap. Chemotherapy was administered 4 times along with 5,040 cGY of radiotherapy. (A) Preoperative view. (B) Post-adjuvant therapy at the 18-month follow-up. There were no complications after radiotherapy. CTx, chemotherapy; $R T x$, radiotherapy.
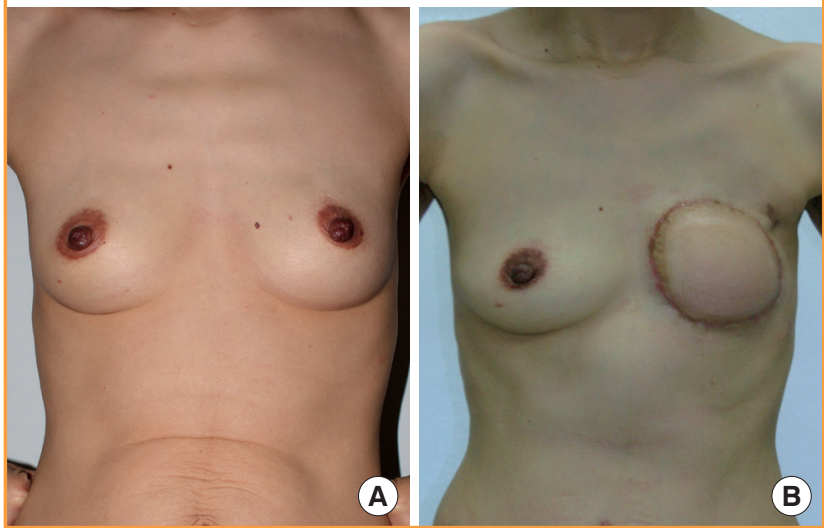

Fig. 4. Redness after adjuvant therapy with CTx+RTx

A 53-year-old woman who underwent breast reconstruction with latissimus dorsi pedicled flap. Chemotherapy and 6,040 cGY of radiotherapy were administered 4 times. (A) Immediate postoperative view. (B) Post-adjuvant therapy at the 2-year follow-up. CTx, chemotherapy; $R T x$, radiotherapy.
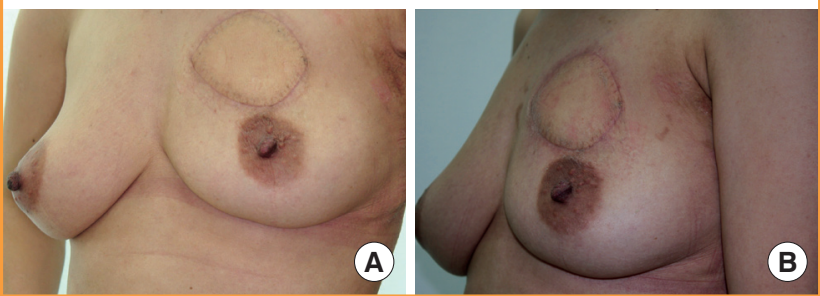

1 (0.4\%), flap contraction (Fig. 6); and there was no flap necrosis or loss.

The complications that occurred in each group are presented in a Table 4. For each complication that occurred in each group, there were no significant differences in the incidence of the complications $(P>0.05)$. Most of the complications that occurred disappeared within 6 months after adjuvant therapy. The group that did not receive postoperative adjuvant therapy did not experience complications. The BREAST-Q patient satisfaction survey showed the group that did not receive adjunctive therapy scored 3.25 points, the group that received adjunctive therapy after immediate breast reconstruction scored 3.11 points, and the group that underwent delayed breast reconstruction scored 3.15 points (Table 5 ). Therefore, satisfactory results of the operations were achieved as shown by the patient satisfaction scores.

\section{Fig. 5. Hyperpigmentation after adjuvant therapy with RTx}

A 51-year-old woman who underwent latissimus dorsi pedicled flap. Only radiotherapy was applied. The radiation dose was 6,040 cGY. (A) Immediate postoperative view. (B) Post-adjuvant therapy at 2 months follow-up. Mild pigmentation around the wound margin. RTx, radiotherapy.

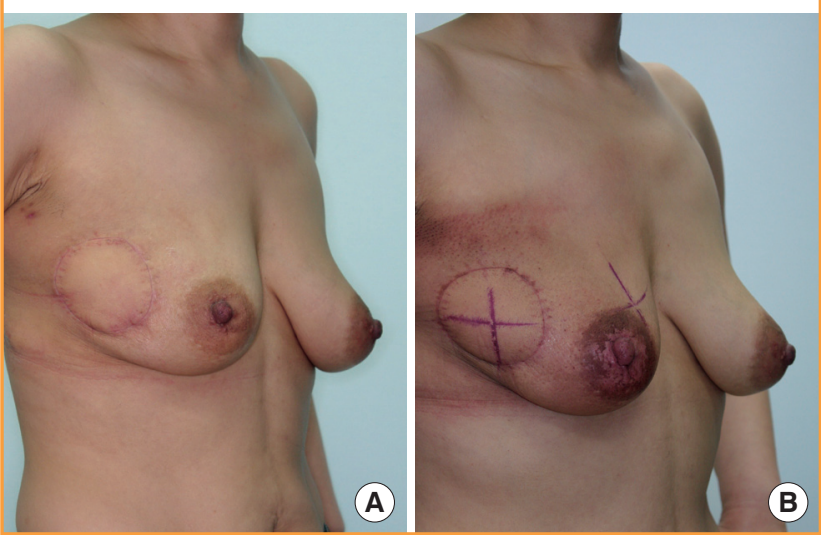

Fig. 6. Flap contracture after adjuvant therapy with RTx

A 55-year-old woman underwent transverse rectus abdominis myocutaneous pedicled flap, chemotherapy, and 5,040 cGY of radiotherapy. (A) Post-adjuvant therapy at 4 months follow-up. (B) Closed view. RTx radiotherapy.
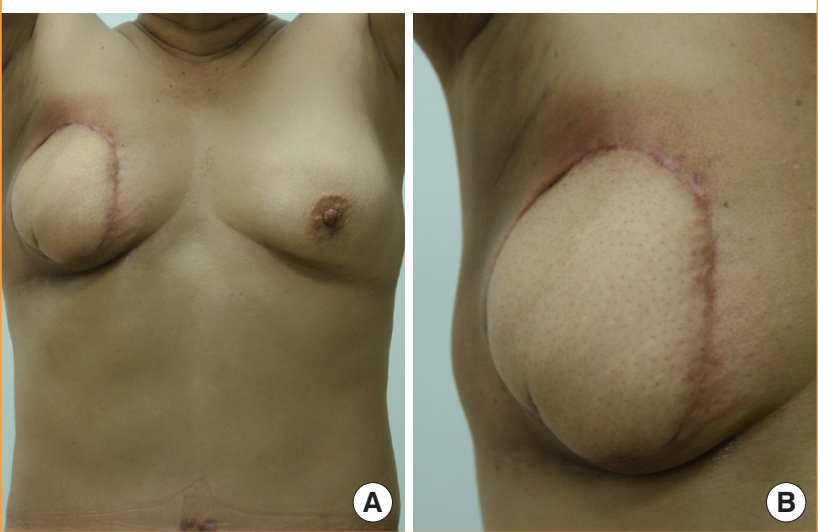

Table 4. Complications that occurred in each group

\begin{tabular}{|lccccc|}
\hline & CTx+RTx & CTx & RTx & None & $\begin{array}{c}\text { P- } \\
\text { value }^{\text {a) }}\end{array}$ \\
\hline Redness & 1 & 0 & 3 & 0 & 0.285 \\
Swelling & 2 & 0 & 2 & 0 & 0.821 \\
Hardness & 2 & 0 & 3 & 0 & 0.528 \\
Hyperpigmentation & 2 & 0 & 2 & 0 & 0.864 \\
Bullae & 0 & 0 & 1 & 0 & 0.227 \\
Fat necrosis & 2 & 2 & 0 & 0 & 0.120 \\
Flap contracture & 1 & 0 & 0 & 0 & 1 \\
Total & 10 & 2 & 9 & 0 & 0.119 \\
\hline $\begin{array}{l}\text { CTx, chemotherapy; RTx, radiotherapy. } \\
\text { a)Fisher's chi-squared test. }\end{array}$ & & & & \\
\hline
\end{tabular}


Table 5. Satisfaction score according to the adjuvant therapy and reconstructive surgery time

\begin{tabular}{|lc|}
\hline & Satisfaction score \\
\hline Immediate + no adjuvant Tx & 3.25 \\
Immediate + adjuvant Tx & 3.11 \\
Delayed (only TRAM) & 3.15 \\
\hline Tx, Treatment; TRAM, transverse rectus abdominis myocutaneous. \\
\hline
\end{tabular}

\section{DISCUSSION}

With the increase in early detection of breast cancer due to the advances in diagnostic methods such as ultrasonography, computed tomography (CT), and MRI, the known incidence of breast cancer in Korean women after uterine cancer and stomach cancer has increased. Due to this, mastectomies are frequently performed, and thus, more breast reconstruction procedures are also carried out. It has been shown in numerous patients that breast reconstructive surgery provides significant help in overcoming cancer [1]. Breast reconstruction can be broadly classified into immediate breast reconstruction and delayed breast reconstruction according to the reconstruction period. Immediate breast reconstruction has the advantage of preventing fear and mental suffering from the changes in the body after mastectomy because the patient does not see the results of the mastectomy. It also has the advantages of better cosmetic results due to the absence of scars and the non-exposure of blood vessels upon mastectomy as well as decreasing the medical costs by reducing the number of surgeries through simultaneously performing the mastectomy and breast reconstruction. Immediate breast reconstruction, however, also has drawbacks such as the uncertainty of the progress of the treatment after the mastectomy, and the psychological burden of the surgeon who has to reduce the time for shaping the reconstructed breast due to the need to perform the reconstructive surgery during the mastectomy [6].

On the other hand, delayed breast reconstruction provides time for physical recovery after mastectomy, and makes it easier to verify the complete recovery by observing the progress of the breast cancer treatment. It has the disadvantages, however, of requiring more frequent surgeries and requiring breast reconstruction using autologous tissue due to the extreme lack of flexibility because of the cicatricial contracture.

Additionally, according to Wellisch et al. [7], 60\% of patients who have undergone delayed breast reconstruction have shown a mentally hypersensitive reaction that included obsessive-compulsive reaction, hypersensitivity in personal relations, depression, hostility, paranoia, and rage. Even in this study, 12 (75\%) of the 16 patients who underwent delayed breast reconstruc- tion showed mentally hypersensitive reactions, though there were differences in the severity of their reactions after surgery. However, only 21 (10\%) of the 204 patients who underwent immediate breast reconstruction complained of a sense of loss or depression after the surgery. Therefore, immediate breast reconstruction can also be deemed superior with regard to these mental impacts. Hence, despite the advantages of delayed breast reconstruction, the number of cases of immediate breast reconstruction is increasing due to its cosmetic, mental, and economic advantages.

Numerous studies have shown that breast reconstruction using autologous tissue has resulted in a lower incidence of complications after postoperative adjunctive therapy and superior cosmetic results compared to breast reconstruction using a prosthesis [2-4]; moreover, in the authors' hospital, a method that employs autologous tissue was used when performing breast reconstruction. Among the autoplasty methods, reconstructive surgery using LD pedicled flap or TRAM flap was performed. The LD pedicled flap was used extensively for local excisions and when the breast on the opposite side was not too large. The reconstruction with TRAM flap was used after total mastectomy and when the breast on the tendon side was relatively big. Up to the present, approximately 200 random clinical trials have been performed for postoperative adjuvant therapy, among which many of these studies carried out follow-up observations of patients for over 20 years and have come up with a definite conclusion on the treatment results. Unlike in the past, not only has adjuvant therapy after mastectomy been mostly performed in the recent stage, but also in the initial stages; and it has been developed because it prevents locoregional recurrence of breast cancer and enhances the average survival rate $[8,9]$. Despite these advantages, however, it may cause various complications such as delayed healing, skin necrosis, discoloration, fat necrosis, flap necrosis, flap contraction, flap contracture, flap deformation, flap hardening, infection, and inflammation.

According to a recently announced thesis, a group that had received adjuvant therapy after immediate breast reconstruction using autologous tissue had a high incidence of complications, but its patient satisfaction did not significantly differ from that of the group that had not received adjuvant therapy. Moreover, the incidence of complications in the group that received adjuvant therapy after immediate breast reconstruction using autologous tissue was slightly higher than that in the group that had undergone delayed breast reconstruction, but the first group showed superior results in patient satisfaction [4]. Similar results were achieved in this study. There was no incidence of complications in the group that did not undergo adjuvant therapy, and it scored 3.25 points in patient satisfaction. In the group that underwent 
adjuvant therapy after immediate breast reconstruction, complications occurred in 21 (11.4\%) of the total of 183 patients, and the score for patient satisfaction was 3.11 points. In the group of patients who underwent delayed breast reconstruction, the patient satisfaction score was 3.15 points.

The analysis of the results of this study showed that the group that had undergone adjuvant therapy after immediate breast reconstruction had a high incidence of complications, which did not significantly differ, however, from that of the patient group that did not undergo adjuvant therapy. Moreover, the former group showed no incidence of major complications related to flaps, and among these, even most of the complications that occurred were observed to have disappeared within 6 months after adjuvant therapy. From the perspective of patient satisfaction, there was no significant difference $(\mathrm{P}>0.05)$ compared to the group that did not receive adjuvant therapy and the group that underwent delayed breast reconstruction.

Such findings might have resulted from the technological advances in radiotherapy and the development of surgical methods.

The development of the medical linear accelerator in the 1950s, the performance of 3D therapy design, and irradiation using computers since the late 1990s have reduced the side effects of radiation and have made it possible to perform more effective radiotherapy by irradiating more precisely, that is, only irradiating the cancerous tissue. Moreover, 5,040-6,040 cGy of irradiation is divided and irradiated in 28 to 33 smaller doses. This method, which is effective against tumors and minimally damages the flaps, can also reduce radiation-induced side effects of the flaps due.

According to numerous studies, various complications may arise depending on the length of time after the irradiation. In the initial stages of the disease, these possible complications include breast edema, fat necrosis, calcification, radiation-induced tuberculosis, and pleural effusion; and in the intermediate stages, breast fibrosis, contraction of the mammary glands, difficulty in breastfeeding, fracture of the irradiated area, pulmonary fibrosis, and pericardial disease. In the latter stages, cardiac disorder or a radiation-induced malignant tumor may occur [10-12]. Viewing the effects of radiotherapy from the perspective that it reduces the locoregional recurrence of a postoperative primary tumor and further improves the survival rate, it will become a meaningless medical procedure if it does not help enhance the final survival rate due to the occurrence of various complications after radiotherapy. Therefore, the exposure dose of 5,040$6,040 \mathrm{cGy}$ investigated by the authors is the dose that can minimize complications while reducing the locoregional recurrence of the postoperative primary site, and it is the exposure dose that can effectively improve the final survival rate. Because contact radiography using photon and electron beams, and 3D therapy technology that performs more precise and accurate irradiation while searching for the target, are now being developed, more effective radiotherapy will be performed in the future. Even surgical procedures will become more effective due to technological advances such as those that cause minimal damage to the flaps and the flap pedicle. In addition to the gradual development of radiotherapy and surgical methods, efforts should be made to ensure further flap stabilization and more satisfactory patient results even after the performance of adjuvant therapy following immediate breast reconstruction.

In sum, 183 patients who underwent adjuvant therapy after breast reconstruction using autologous tissue at the authors' hospital showed no significant difference in complications, in contrast to the patient group that did not undergo adjuvant therapy after immediate breast reconstruction; and the patients who underwent adjuvant therapy showed no incidence of major complications related to flaps. Even among the complications that arose, most were observed to have disappeared. Furthermore, the authors report that the immediate breast reconstruction using autologous tissue ensured flap stability and yielded good patient satisfaction results even after postoperative chemotherapy and radiotherapy. It also showed that immediate reconstruction is safe and useful for patients who need postoperative adjuvant therapy.

\section{REFERENCES}

1. Alderman AK, Collins ED, Schott A, et al. The impact of breast reconstruction on the delivery of chemotherapy. Cancer 2010;116:1791-800.

2. Spear SL, Ducic I, Low M, et al. The effect of radiation on pedicled TRAM flap breast reconstruction: outcomes and implications. Plast Reconstr Surg 2005;115:84-95.

3. Kronowitz SJ, Robb GL. Radiation therapy and breast reconstruction: a critical review of the literature. Plast Reconstr Surg 2009; 124:395-408.

4. Lee BT, T AA, Colakoglu S, et al. Postmastectomy radiation therapy and breast reconstruction: an analysis of complications and patient satisfaction. Ann Plast Surg 2010;64:679-83.

5. Jagsi R, Abrahamse P, Morrow M, et al. Postmastectomy radiotherapy for breast cancer: patterns, correlates, communication, and insights into the decision process. Cancer 2009;115:1185-93.

6. Paulson RL, Chang FC, Helmer SD. Kansas surgeons' attitudes toward immediate breast reconstruction: a statewide survey. Am J Surg 1994;168:543-6.

7. Wellisch DK, Schain WS, Noone RB, et al. Psychosocial 
correlates of immediate versus delayed reconstruction of the breast. Plast Reconstr Surg 1985;76:713-8.

8. Zhang YJ, Sun GQ, Chen J, et al. Postmastectomy locoregional recurrence and survival in early stage breast cancer patients with one to three axillary lymph node metastases. Ai Zheng 2009;28:395-401.

9. Buchholz TA, Strom EA, Perkins GH, et al. Controversies regarding the use of radiation after mastectomy in breast cancer. Oncologist 2002;7:539-46.

10. Yi A, Kim HH, Shin HJ, et al. Radiation-induced complica- tions after breast cancer radiation therapy: a pictorial review of multimodality imaging findings. Korean J Radiol 2009; 10:496-507.

11. Senkus-Konefka E, Jassem J. Complications of breast-cancer radiotherapy. Clin Oncol (R Coll Radiol) 2006;18:229-35.

12. Senkus-Konefka E, Welnicka-Jaskiewicz M, Jaskiewicz J, et al. Radiotherapy for breast cancer in patients undergoing breast reconstruction or augmentation. Cancer Treat Rev 2004;30:671-82. 